\section{Optimal design of single resonant and ultrabroadband long-period fiber grating filters}

\author{
Cheng-Ling Lee ${ }^{a}$ and Pin Han ${ }^{b}$ \\ ${ }^{a}$ National United University, Department of Electro-Optical \\ Engineering, No. 1 Lien-Da, Kung-Ching Li, Miaoli, \\ 360 Taiwan \\ ${ }^{\mathrm{b}}$ National Chung Hsing University, Institute of Precision \\ Engineering, 250 Kuo Kuang Road, Taichung 402, Taiwan \\ E-mail: cherry@nuu.edu.tw
}

Abstract. We propose a spectral flat-top, single resonant and ultrabroadband-more than $180 \mathrm{~nm}$ in a $-20-\mathrm{dB}$ bandwidth-long-period fiber grating (LPG) filter. The ultrabroadband LPG is based on a thin cladding layer LPG synthesized by the Lagrange multiplier optimization (LMO) algorithm. As the bandwidth and resonant spectra cover a very wide band, both material dispersion and waveguide dispersion were included in the calculations of the LMO method. To the best of our knowledge, the bandwidth of the designed flat-top LPG filter in the -20-dB coupling is the broadest currently existing in the literature. Such designed LPG devices can be very useful for a variety of applications in broadband optical communication systems. () 2009 Society of Photo-Optical Instrumentation Engineers. [DOI: 10.1117/1.3194285]

Subject terms: ultrauroadband long-period fiber grating; lagrange multiplier optimization; grating synthesis; bandwidth; dispersion.

Paper 090172LR received Mar. 12, 2009; revised manuscript received Jun. 5, 2009; accepted for publication Jun. 12, 2009; published online Aug. 7, 2009.

\section{Introduction}

Long-period fiber gratings (LPGs), coupled with a guided core mode in copropagating modes in an optical fiber, have been widely used as band-rejection filters, wavelengthdependent loss filters, mode converters, and high-sensitivity sensors. ${ }^{1}$ Recently, due to the rapid development of ultrawideband $\left(\mathrm{S}^{+}\right.$band-L-band) gain amplifiers, a broadband gain flattening device within a very wide bandwidth is needed; consequently, the LPGs appear to be good candidates for gain flattening applications. ${ }^{2}$ However, broadband LPG filters could lead to a high insertion loss as a result of the high-order cladding modes coupling in a typical LPG device. To overcome this shortcoming, Jeong and Oh theoretically presented a new LPG filter that significantly enhanced the free spectral range (FSR) by controlling the waveguide dispersion of the cladding modes in fluorinedoped-cladding LPG. ${ }^{3}$ Their model further controlled material dispersion in the cladding region using doped ions $\mathrm{Cr}^{3+}$ to change the effective indices of the cladding modes, ${ }^{4}$ thereby changing the phase-matching conditions of LPGs as well. However, the proposed methods are not very practical due to the highly difficult fabrications of such special fibers.

Another technique to enhance the FSR of LPG involves

0091-3286/2009/\$25.00 @ 2009 SPIE etching the fiber cladding with hydrofluoric acid (HF). Yin et al. demonstrated that an HF-etched ultrathin LPG (with a cladding thickness of $28 \mathrm{~mm}$ ) with first-order cladding mode coupling provides a wide tuning range of $120 \mathrm{~nm}$ and has only a single resonant peak over a wide wavelength range (1000-2000 nm). ${ }^{5}$ These authors subsequently presented a highly sensitivity sensor based on an ultrathin-clad LPG, with high-order mode coupling that can be tuned for $>220 \mathrm{~nm}$ as well as a single resonant peak in a wide range of wavelength bands; ${ }^{6}$ however, the inherent bandwidth of the resonance peaks of LPGs are narrow, at about several nanometers, because the phase-matching condition between the two optical modes is satisfied at only a specific resonant wavelength. Indeed, this is the major drawback of the bandwidth-versus-coupling strength trade-offs for practical applications when using such a narrowband LPG device.

To enhance LPGs' performance for broadband resonance of LPG, Wang and Ramachandran et al. ${ }^{7-9}$ demonstrated several broadband, spectrally flat LPG-based devices in especially designed few-mode fibers, which were designed to have two coupled modes with identical group velocities in the desired spectral range of wavelength. The LPG coupling in this condition can achieve a broadband mode conversion because it occurs in the turnaround point (TAP) between the fundamental mode and a specific higher-order guided core mode (with negative chromatic dispersion). Although engineering a fiber waveguide to have identical group velocities for two modes may yield a better broadband grating if a LPG is written to couple at the TAP, the bandwidth of the proposed LPG devices cannot be $>180 \mathrm{~nm}$ at the $-20-\mathrm{dB}$ coupling strength (the record bandwidth is $110 \mathrm{~nm}$ with a flatband for the variable optical attenuator in Ref. 9). Moreover, the special dispersiontailor fiber must be difficultly fabricated using a specially designed process.

The current paper presents another option: a spectral flat-top, single resonant, and ultrabroadband LPG (UBLPG) with a bandwidth of $>180 \mathrm{~nm}$ at $-20-\mathrm{dB}$. The proposed device is based on a thin-cladding LPG with synthesizing using the Lagrange multiplier optimization (LMO) algorithm. To the best of our knowledge, the bandwidth of the designed UBLPG at $-20 \mathrm{~dB}$ is the broadest in the existing literature. Such devices can be utilized as broadband mode converters and broadband couplers for broadband optical applications. They can also be used as a broadband all-fiber polarizer when the UBLPG is written in the birefringent fiber. Moreover, due to core and cladding coupling in the thin-clad LPG, these devices could be used as a tunable broadband filter by changing the refractive index of the surrounding. The current study examines the waveguide dispersion as well as material dispersion, calculated using the Sellmeier equation, involved in the numerical analysis for covering such a wideband wavelength range. The effective indices of the guided modes are subsequently obtained by solving the characteristic equations of the fiber. From the simulation results, the bandwidth of the resonant peak for the designed LPG can be substantially broadened when using the proposed LMO algorithm, which is a variationbased approach that has been proven to be very effective in designing different fiber grating filters for the applications of fiber optics. ${ }^{10-12}$ Furthermore, the presented UBLPG device has only a single resonant peak over a very wide 
Table 1 Sellmeier coefficients of silica glass doped with $\mathrm{GeO}_{2} \cdot{ }^{14}$

\begin{tabular}{ccc}
\hline \hline & $\mathrm{SiO}_{2}(100 \mathrm{~mol} \%)$ & $\mathrm{SiO}_{2}(96.5 \mathrm{~mol} \%)$ \\
$\mathrm{GeO}_{2}(0 \mathrm{~mol} \%)$ & 0.7004080 \\
$\mathrm{~A}_{1}$ & 0.6961663 & 0.0684241 \\
$\mathrm{~B}_{1}$ & 0.0684043 & 0.4188000 \\
$\mathrm{~A}_{2}$ & 0.4079426 & 0.1175610 \\
$\mathrm{~B}_{2}$ & 0.1162414 & 0.8959635 \\
$\mathrm{~A}_{3}$ & 0.8974794 & 9.9642629 \\
$\mathrm{~B}_{3}$ & 9.8961610 & \\
\hline \hline
\end{tabular}

wavelength range $(>600 \mathrm{~nm})$. We believe that such UBLPG devices will be very useful in a variety of applications for an ultrawideband optical communications to cover the $S^{+}-L$ bands.

\section{LMO Algorithm for UBLPG}

LPGs incorporate coupling guided through the core mode into the forward propagating modes in a fiber by phase matching the two modes at resonant wavelengths. LPGs characteristics can be effectively identified using the coupled mode equations. ${ }^{13}$ As the single resonance peak of the designed UBLPG is extended over an extremely wide wavelength range, the material dispersion should be considered in the simulation processes. The dispersive characteristics of pure silica $\left(\mathrm{SiO}_{2}\right)$ and silica doped with dopants such as $\mathrm{GeO}_{2}$ have been precisely calculated using the following Sellmeier equation:

$n^{2}-1=\sum_{i=1}^{3} \frac{A_{i} \lambda^{2}}{\lambda^{2}-B_{i}^{2}}$,

where $A_{i}$ and $B_{i}$ are Sellmeier coefficients and $\lambda$ is the wavelength, measured in microns. The silica doped with $\mathrm{GeO}_{2}$ used in this designed case is shown in Table $1 .{ }^{14}$ The effective index of the guided core mode can thus be obtained using dispersion relations. ${ }^{13}$ The effective indices of cladding modes can be determined by solving the dispersion relations of three-layer optical fibers. ${ }^{6}$ Once the effective core and cladding modes are calculated, the resonant wavelengths of the LPGs can be expressed as $\lambda_{p}^{m}=\left(n_{\text {eff-co }}\right.$ $\left.-n_{\text {eff-cl }}^{m}\right) \cdot \Lambda$, where $n_{\text {eff-co }}$ and $n_{\text {eff-cl }}^{m}$ are the effective indices of the core and the m-order cladding modes and $\Lambda$ is the grating period of UBLPG. The detailed theoretical analysis of LMO for synthesized LPGs has been described in a previous work, ${ }^{11}$ in which the boundary conditions for the coupled equations of the Lagrange multipliers were obtained by using the variational method with the cost functional with respect to the parameters of the designed LPG. In the current study, to improve the target performance, the corresponding boundary conditions for Lagrange multipliers coupled equations at $L$ are strategically calculated in decibel scale, as follows:

$\mu^{\mathrm{co}}(L)=-2 \cdot A^{\mathrm{co}}(L) \cdot\left(\Delta_{t-d B}\right)$,

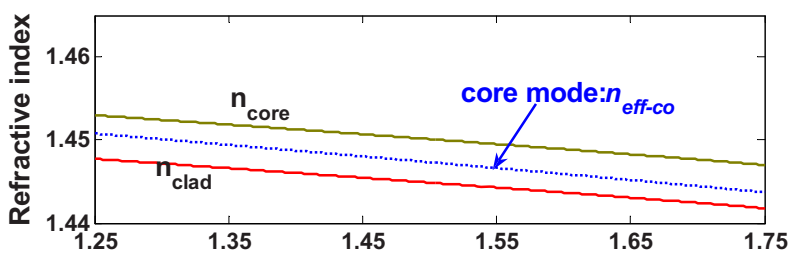

(a)

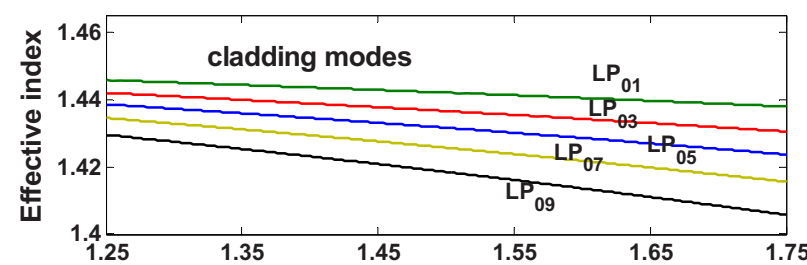

(b)

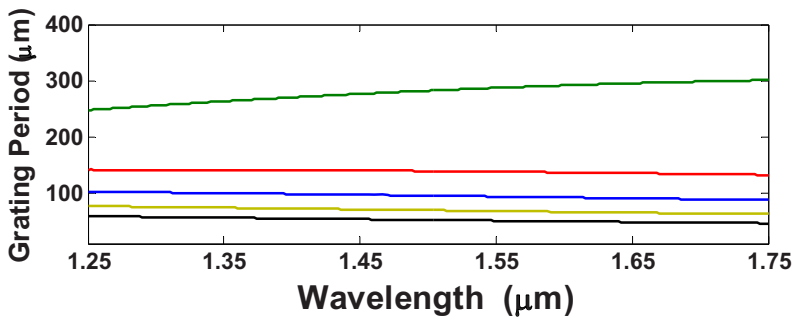

(c)

Fig. 1 (a) Refractive indices of core and cladding of the fiber; the dotted line is the effective index of the guided core mode. (b) The effective indices of the first five odd-cladding modes ( $\mathrm{LP}_{0 m}, m$ $=1,3,5 \ldots$ ) of the proposed fiber device. (c) The phase-matching grating periods versus the coupling wavelengths for the first five odd-cladding modes.

$\mu^{\mathrm{cl}}(L)=0$,

where $\mu^{\mathrm{co}}(L)$ and $\mu^{\mathrm{cl}}(L)$ are the Lagrange multipliers fields for the core and cladding mode of UBLPG at $L, L$ is the grating length, $A^{\mathrm{co}}(L)$ is the mode field of the core mode of the designed UBLPG at $L$, and $\Delta_{t-\mathrm{dB}}=\left\{10 \log _{10}\left[T_{\mathrm{co}}(\lambda)\right]\right.$ $\left.-10 \log _{10}\left[T_{d}(\lambda)\right]\right\}$ is the discrepancy between the output $\left[T_{\text {co }}(\lambda)\right]$ and target transmission spectra $\left[T_{d}(\lambda)\right]$ in the decibel scale. On the basis of the numerical results, it is found that such a strategy using the decibel scale in the formulation of boundary condition (BC) of Lagrange multipliers equations provides a much better improvement in the convergence of the LMO algorithm (decibel-scale LMO) than those cases the $\mathrm{BC}$ are calculated in linear scale (linearscale LMO).

\section{Design Results and Discussion}

This section demonstrates an ultrabroadband thin-clad LPG filter with a single resonant peak in an extremely wide range of wavelength band $(\sim 600 \mathrm{~nm})$ optimally designed using a decibel-scale LMO algorithm. In the analysis, the fiber core was assumed to be germanosilicate glass (with doped $3.5 \mathrm{~mol} \% \mathrm{GeO}_{2}$ ), as in conventional single-mode fibers. The refractive index of cladding was the pure silica, and the surrounding material was air (with a refractive index of 1). The dispersive characteristics of core and cladding were calculated, and the refractive indices are provided in Fig. 1(a). When the fiber core and cladding 


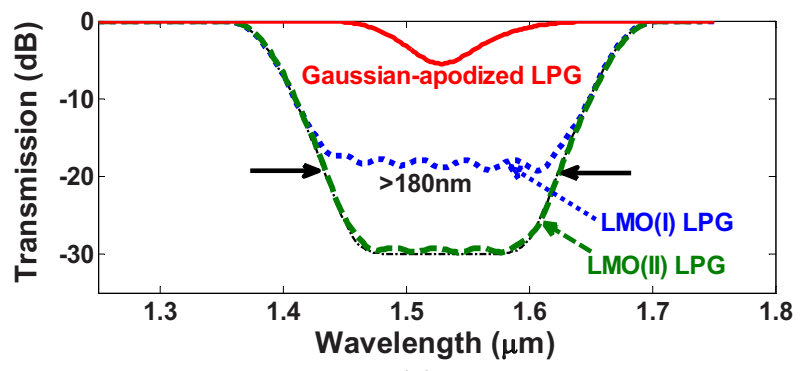

(a)

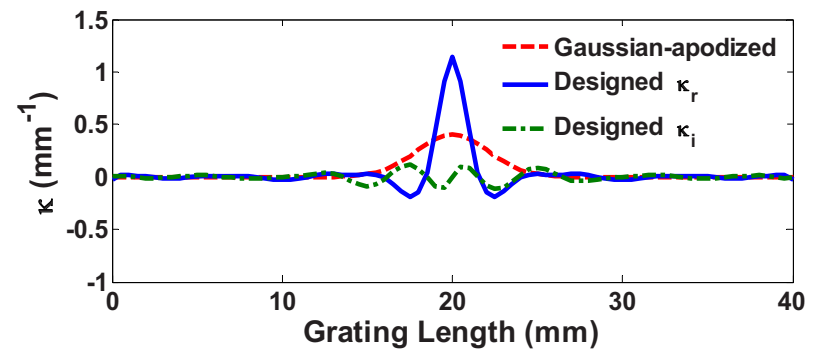

(b)

Fig. 2 (a) Transmission spectra of the designed UBLPGs. The dotted line is LMO(I) method using linear scale, and the dashed line is LMO(II) method with decibel scale. (b). Real $\left(\kappa_{r}\right)$ and imaginary $\left(\kappa_{i}\right)$ parts of the designed coupling coefficients of UBLPG, with a grating length of $4 \mathrm{~cm}$.

diameters are set at 8.2 and $40 \mu \mathrm{m}$ of the thin-clad LPG, respectively, the effective refractive index of the fundamental core mode can be obtained through the dispersion relation and shown in dotted line of Fig. 1(a). The first five odd-cladding modes determined by the dispersion relations of the three-layer optical fiber ${ }^{6}$ are shown in Fig. 1(b).

From the LPG's phase-matched conditions of LPG, the grating period $(\Lambda)$ can be calculated as a function of the wavelength $(\lambda)$, as shown in Fig. 1(c) for the first five oddcladding mode couplings. If the grating period is set to $285 \mu \mathrm{m}$, the corresponding resonant wavelength $\lambda_{p}$ is $\sim 1.529 \mu \mathrm{m}$ when the core mode and first odd-cladding mode satisfy the matching coupling phase. (One can also choose high-order mode coupling for simulations.) It can be clearly seen that only one resonant peak in a very wide wavelength band emerges, regardless of which cladding mode of coupling is used in the thin-clad LPG. Figure 2(a) depicts the corresponding transmission spectra of nonoptimized (Gaussian apodized) and LMO-UBLPG with a grating length of $40 \mathrm{~mm}$. The coupling coefficients $(\kappa)$ of the designed UBLPGs are shown in Fig. 2(b). The dashed line is the Gaussian apodized, while the solid line and dashed dotted line present real $\left(\kappa_{\mathrm{r}}\right)$ and imaginary $\left(\kappa_{\mathrm{i}}\right)$ parts of the coupling coefficient synthesized by decibel-scale LMO algorithm. From the simulation results of the dotted line of Fig. 2(a), the bandwidth of the designed LMO(I)-LPG can be significantly expanded by using the linear-scale LMO algorithm in Ref. 11. However, the coupling strength of the LMO(I)-LPG is only $-20 \mathrm{~dB}$ for the final convergence because the boundary conditions $\mu^{\mathrm{co}}(L)$ and $\mu^{\mathrm{cl}}(L)$ of the linear-scale LMO algorithm are saturated with the error of $\sim 0.01$.

To improve the performance of the proposed filter when the target performance is in the decibel scale, the decibel- scale LMO algorithm mentioned in the previous section is utilized. The designed LPG [called LMO(II)-LPG] is depicted by the dashed line in Fig. 2(a), which indicates that the almost $-30 \mathrm{~dB}$ coupling strength can be achieved with a bandwidth of about $100 \mathrm{~nm}$ and $-20 \mathrm{~dB}$ with a bandwidth of $180 \mathrm{~nm}$. In addition, these designed LPGs should be polarization insensitive due to the spatially symmetric modes in the cylindrical structure of optical fiber. Furthermore, the LPGs are in general significantly easier to fabricate than FBGs due to the LPG periods, which are hundreds of microns. One can use the UV-beam-scanning point-by-point exposure techniques ${ }^{15}$ to achieve a complex coupling coefficient of the gratings for practical fabrication.

\section{Conclusion}

This paper presented the optimization of a spectral flat-top, ultrabroadband thin-clad LPG filter with a single resonant peak in a very wide range of wavelength band $(\sim 450 \mathrm{~nm})$ using the decibel-scale LMO algorithm. For the first time, the spectrum in the $-20-\mathrm{dB}$ bandwidth of the designed UBLPG filter can be broadened beyond $180 \mathrm{~nm}$ by using the proposed of LMO method. This proposed UBLPG also can be utilized as a broadband polarizer when the designed LPG is written in an elliptical core fiber or a birefringent fiber. We believe such a novel UBLPG device will be very useful in applications for ultrabroadband fiberoptics technology.

\section{References}

1. A. M. Vengsarkar, P. J. Lemaire, J. B. Judkins, V. Bhatia, T. Erdogan, and J. E. Sipe, "Long-period fiber gratings as band rejection filters," J. Lightwave Technol. 14, 58-65 (1996).

2. A. M. Vengsarkar, J. R. Pedrazzani, J. B. Judkins, P. J. Lemaire, N. S. Bergano, and C. R. Davidson, "Long-period fiber-grating-based gain equalizers," Opt. Lett. 21, 336-338 (1996).

3. H. Jeong and K. Oh, "Theoretical analysis of cladding-mode waveguide dispersion and its effects on the spectra of long-period fiber grating," J. Lightwave Technol. 21, 1838-1845 (2003).

4. H. Jeong and K. Oh, "Enhancement of free spectral range of the resonance peaks in a long-period fiber grating by controlling material dispersion of cladding modes," Opt. Commun. 199, 103-110 (2001).

5. S. Yin, K.-W. Chung, and X. Zhu, "A novel all-optic tunable longperiod grating using a unique double-cladding layer," Opt. Commun. 196, 181-186 (2001).

6. K.-W. Chung and S. Yin, "Analysis of a widely tunable long-period grating by use of an ultrathin cladding layer and higher-order cladding mode coupling," Opt. Lett. 29, 812-814 (2004).

7. Z. Wang and S. Ramachandran, "Ultrasensitive long-period fiber gratings for broadband modulators and sensors," Opt. Lett. 28, 2458 $2460(2003)$

8. S. Ramachandran, Z. Wang, and M. Yan, "Bandwidth control of longperiod grating-based mode converters in few-mode fibers," Opt. Lett. 27, 698-700 (2002).

9. S. Ramachandran, M. Yan, E. Monberg, F. V. Dimarcello, P. Wisk, and S. Ghalmi, "Record bandwidth, spectrally flat coupling with microbend gratings in dispersion-tailored fibers," IEEE Photon. Technol. Lett. 15, 1561-1563 (2003).

10. C. L. Lee, R. K. Lee, and Y. M. Kao, "Design of multichannel DWDM fiber Bragg grating filters by Lagrange multiplier constrained optimization," Opt. Express 14, 11002-11011 (2006).

11. C. L. Lee, R. K. Lee, and Y. M. Kao, "Synthesis of long-period fiber gratings with a Lagrange multiplier optimization method," Opt. Commun. 281, 61-74 (2008)

12. C. L. Lee, R. K. Lee, and Y. M. Kao, "Lagrange multiplier constrained optimization for designing narrowband dispersionless fiber bragg gratings," Opt. Eng. 47, 015005 (2008).

13. T. Erdogan, "Fiber grating spectra," J. Lightwave Technol. 15, $1277-$ 1294 (1997).

14. J. W. Fleming, "Dispersion in $\mathrm{GeO}_{2}-\mathrm{SiO}_{2}$ glasses," Appl. Opt. 23(24), 4486-4493 (1984).

15. A. I. Kalachev, D. N. Nikogosyan, and G. Brambilla, "Long-period fiber grating fabrication by high-intensity femtosecond pulses at 211 nm," J. Lightwave Technol. 23, 2568-2578 (2005). 